\title{
The NRL Multi Aperture SAR System
}

\author{
Mark Sletten*, Steven Menk*, Jakov Toporkov*, Robert W. Jansen* and Luke Rosenberg ${ }^{\dagger \ddagger}$ \\ * US Naval Research Laboratory, \\ ${ }^{\dagger}$ Defence Science and Technology Organisation, ${ }^{\ddagger}$ University of Adelaide, Australia. \\ Email: mark.sletten@nrl.navy.mil
}

\begin{abstract}
This paper describes the Naval Research Laboratory Multi Aperture Synthetic Aperture Radar (NRL MSAR) and presents initial results from the first field deployment of this system. The NRL MSAR is an airborne test bed designed to investigate remote sensing and surveillance applications that exploit multiple along-track phase centers, in particular, applications that require measurement of scene motion. The system operates at $\mathrm{X}$ band and supports 32 along-track phase centers through the use of two transmit horns and 16 receive antennas. As illustrated in this paper, SAR images generated with these phase centers can be coherently combined to directly measure scene motion using the Velocity SAR (VSAR) algorithm. In September 2014, this unique radar was deployed for the first time on an airborne platform, a Saab 340 aircraft. This paper presents a description of the system, initial images from the September 2014 tests, and the results of initial coherent analyses to produce estimates of scene and target motion. These images were collected over an ocean inlet and contain a variety of moving backscatter sources, including automobiles, ships, shoaling ocean waves, and tidal currents.
\end{abstract}

\section{INTRODUCTION}

High fidelity imaging of moving targets is a well-known challenge for synthetic aperture radar (SAR), especially in ocean environments. Standard SAR processing methods assume the entire scene is stationary, and interpret the Doppler histories in the data accordingly. Any Doppler shifts introduced by target or scene motion will be misinterpreted and the corresponding backscatter will be misplaced in the image. The classic example of this is the 'train-off-the-track', in which the signature of a range-traveling train appears displaced from the signature of the track upon which it is running. More serious distortions occur if different parts of the target move at different speeds, which is often the case for vessels moving on a dynamic ocean surface, as the target's signature in the image will then be smeared, not merely displaced. Advanced techniques utilizing multiple-aperture SAR (MSAR) systems have been developed to detect moving targets, estimate their velocities, and correct their positions within the image [1][3]. The apertures in these systems are arranged along the flight axis to provide measurements of the scene at slightly different times but from the same vantage point in space, thereby allowing separation of the scene motion from that of the SAR platform. However, all these techniques assume the clutter is stationary, and thus they have limited applicability to ocean environments.

MSAR systems of this type can indeed provide a significant advantage for ocean applications. Rather than simply providing a means to remove stationary clutter, an MSAR with its apertures arranged along the flight direction provides a means to directly measure radial motion at each and every pixel across the entire scene. To the degree this motion can be measured, the image artifacts it produces can then be removed [4], [5].
The NRL MSAR is described in Section II with the preprocessing stages outlined in Section III. The VSAR algorithm is then described in Section IV with the initial results shown in Section V from a test site on the Outer Banks of North Carolina. The final Section VI then describes some enhanced processing techniques to highlight the velocity scatterers of interest.

\section{THE NRL MSAR SYSTEM}

The NRL MSAR is an airborne system based on the NRL Focused Phased Array Imaging Radar (NRL FOPAIR), a ground-based MSAR test bed [5]. The MSAR system operates at X-band with a center frequency of $9.875 \mathrm{GHz}$ and uses linear FM chirped waveforms with a bandwidth of $220 \mathrm{MHz}$ to achieve a range resolution of approximately $0.7 \mathrm{~m}$. The peak radiated power is approximately $1.4 \mathrm{~kW}$, while the aggregate pulse repetition frequency (PRF) of $25 \mathrm{kHz}$ and pulse length of $6 \mu \mathrm{s}$ produce an average power of $210 \mathrm{~W}$. The system flies on a Saab 340 aircraft using a belly-mounted radome with a nominal incidence angle of $70^{\circ}$, as shown in Fig. 1. Typical altitude and airspeeds are $914 \mathrm{~m}(3000 \mathrm{ft})$ and $70 \mathrm{~m} / \mathrm{s}$, respectively.

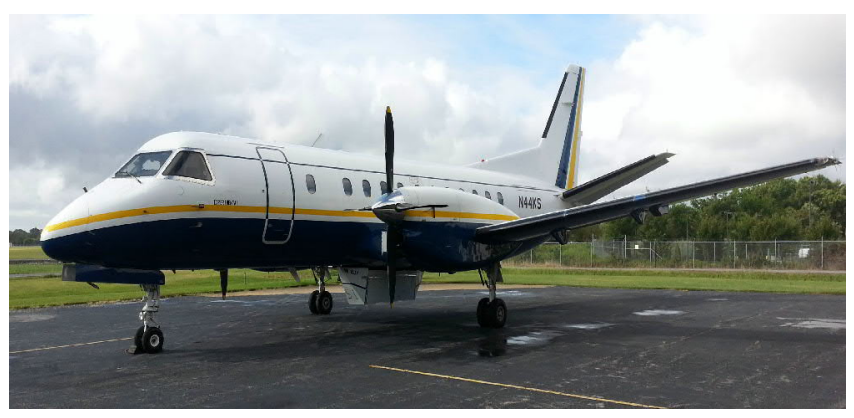

Fig. 1. Saab 340 aircraft with belly mounted radome.

As operated during its inaugural airborne tests in September 2014, the MSAR system uses 16 receive antennas, equally spaced along the flight axis with a separation of $10.48 \mathrm{~cm}$, and two transmit horns, with a spacing of $125.8 \mathrm{~cm}$. A photograph of the antennas as installed in the Saab radome is shown in Fig. 2. The long white enclosures below the two transmit horns contain the 16 receive antennas. All antennas are vertically polarized. During odd-numbered pulse repetition intervals (PRIs), the aft-mounted horn transmits an 'up' chirp, while during even PRIs, the fore-mounted horn radiates a 'down' chirp. During each pair of up-down PRIs, four of the 16 receive antennas are connected to a four-channel receiver and sampled by a high speed data recorder. After each pair of PRIs, a bank of microwave switches is reconfigured to connect 
the next group of four receive antennas to the receiver and data recorder. After a total of 4 up-chirp/down-chirp PRI pairs $(320 \mu \mathrm{s})$, this scan sequence is repeated. In this manner, 32 phase centers corresponding to each combination of transmit and receive antennas are generated and sampled at a perphase-center PRF of $3.125 \mathrm{kHz}$. This PRF is sufficient to allow production of 32 SAR images, one corresponding to each phase center, that are free from azimuthal ambiguities. Since the location of a given phase center is halfway between the corresponding transmit and receive antennas, the alongtrack spacing between these phase centers is $5.24 \mathrm{~cm}$, half the physical spacing between the receive elements. Note that given the spacing between the two transmit horns, four of the phase centers corresponding to the aft-mounted transmit horn are collocated with four produced by the fore-mounted horn. Thus the total number of independent phase centers is 28 with total length of $141.5 \mathrm{~cm}$.

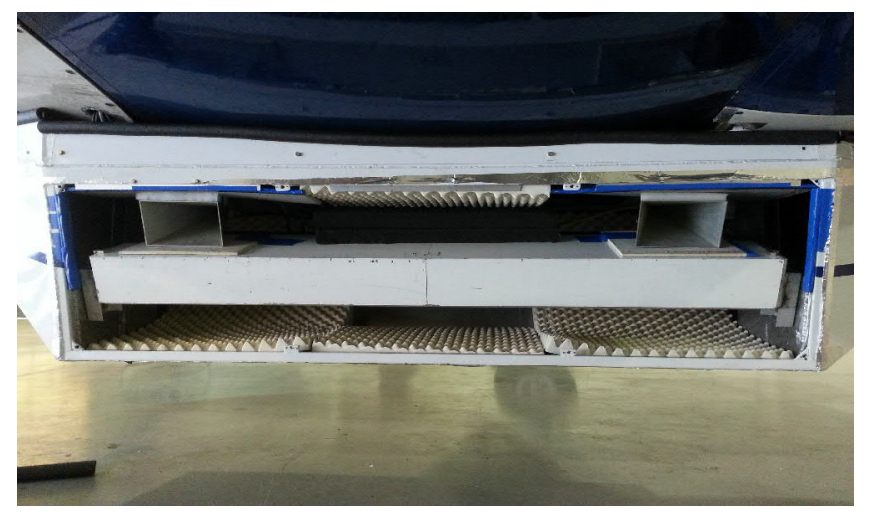

Fig. 2. MSAR in its radome.

The large number of phase centers and the requirement to revisit each frequently enough to allow alias-free SAR imaging produces a high average data rate. During each PRI $(40 \mu \mathrm{s})$, 8192 8-bit samples from each of the 4 data recorder channels are written to a redundant array of independent disks (RAID) for a sustained data rate of $819 \mathrm{MB} / \mathrm{s}$. The recorder is based on a 4-channel, 12-bit acquisition card running at a sample rate of $500 \mathrm{MS} / \mathrm{s}$. Selection of the 8192-sample range window within each PRI and reduction of the 12-bit samples down to 8-bits is performed by a field programmable gate array.

Motion of the antennas is measured by a Novatel inertial navigation system mounted in the Saab radome directly behind the antennas. The Novatel ProPak6 OEM638 receiver blends GPS position data with measurements from a Litton LN200 inertial measurement unit to determine the precise positions of all phase centers at a $100 \mathrm{~Hz}$ rate. These data are used during image formation processing in order to compensate for non-ideal aircraft motion.

\section{PRE-PROCESSING}

Before the VSAR image can be produced, a number of pre-processing steps are required. Firstly, a digital baseband conversion is applied to the data followed by a low pass filter designed to remove any signal artefacts outside the frequency band of interest. A chirp scaling algorithm is then implemented to form a SAR image for each phase centre with extensions designed to motion compensate the resulting image [6]. After the images are formed, a final step is required to balance the phase and magnitude between each phase centre. The phase mismatch comprises a linear term due to the along track displacement of the phase centres and a non-linear term due to the changing aircraft position along the scene. A third nonlinear component of the phase error occurs due to the antenna mismatch and the unbalanced radar hardware. These hardware imbalances also affect the magnitude of the received signal and require calibration using real radar echoes to account for the non-linearity.

The algorithm selected for channel balancing is the 'adaptive 2D calibration' [7], [8]. It was proposed for use in the range frequency / Doppler domain where the bright scatterers appear smoothed out, but can also be applied after image formation. In the azimuth and range spatial frequency domain, the received signal is denoted $Z_{n}\left(k_{x}, k_{y}\right)$ for the $n^{t h}$ phase centre. The channel model is given by

$$
\begin{aligned}
& Z_{1}\left(k_{x}, k_{y}\right) \approx c\left(k_{x}, k_{y}\right) h_{1}\left(k_{y}\right) D_{1}^{\prime}\left(k_{x}\right) \\
& Z_{n}\left(k_{x}, k_{y}\right) \approx c\left(k_{x}, k_{y}\right) h_{n}\left(k_{y}\right) D_{n}^{\prime}\left(k_{x}\right) \exp \left(-j \phi_{n}\left(k_{x}\right)\right)
\end{aligned}
$$

where $c(\cdot)$ is a complex signal common to each channel, $\phi_{n}\left(k_{x}\right)$ is a linear phase shift due to the along track displacement of the phase centre and $h_{n}\left(k_{y}\right)$ and $D_{n}^{\prime}\left(k_{x}\right)$ describe channel specific information along each spatial frequency dimension. The information contained along the range frequency, $h_{n}\left(k_{y}\right)$ is referred to as the 'transfer function', while the azimuth frequency component, $D_{n}^{\prime}\left(k_{x}\right)$ is primarily due to the antenna beampattern which varies between the different channels. The goal of channel balancing is to minimise the channel specific information so the images have a high coherency and similar magnitude. If the phase term $\phi_{n}\left(k_{x}\right)$ is combined into the correction for $D_{n}\left(k_{x}\right)=D_{n}^{\prime}\left(k_{n}\right) \exp \left(-j \phi_{n}\left(k_{x}\right)\right)$, then the ratio between the first and $n^{t h}$ channel is given by

$$
\frac{Z_{1}\left(k_{x}, k_{y}\right)}{Z_{n}\left(k_{x}, k_{y}\right)} \approx \frac{h_{1}\left(k_{y}\right)}{h_{n}\left(k_{y}\right)} \frac{D_{1}\left(k_{x}\right)}{D_{n}\left(k_{x}\right)}=h_{1, n}\left(k_{y}\right) D_{1, n}\left(k_{x}\right) .
$$

The adaptive $2 \mathrm{D}$ calibration algorithm is designed to minimise the square difference between the two terms integrated over the frequency domains. It can be written in terms of the following optimisation [8]:

$$
\begin{aligned}
& \min \\
& \left\{h_{1, n}\left(k_{y}\right), D_{1, n}\left(k_{x}\right)\right\} \int_{k_{x}} \int_{k_{y}} \mid Z_{1}\left(k_{x}, k_{y}\right) \\
& \quad-Z_{n}\left(k_{x}, k y\right) h_{1, n}\left(k_{y}\right) D_{1, n}\left(k_{x}\right) \mid d k_{x} d k_{y}
\end{aligned}
$$

with the solution given as a pair of integral equations

$$
\begin{aligned}
h_{1, n}\left(k_{y}\right) & =\frac{\int_{k_{x}} D_{1, n}^{*}\left(k_{x}\right) Z_{2}^{*}\left(k_{x}, k_{y}\right) Z_{1}\left(k_{x}, k_{y}\right) d k_{x}}{\int_{k_{x}}\left|Z_{2}\left(k_{x}, k_{y}\right) D_{1, n}\left(k_{x}\right)\right|^{2} d k_{x}}, \\
D_{1, n}\left(k_{y}\right) & =\frac{\int_{k_{x}} h_{1, n}^{*}\left(k_{y}\right) Z_{2}^{*}\left(k_{x}, k_{y}\right) Z_{1}\left(k_{x}, k_{y}\right) d k_{y}}{\int_{k_{y}}\left|Z_{2}\left(k_{x}, k_{y}\right) h_{1, n}\left(k_{y}\right)\right|^{2} d k_{y}},
\end{aligned}
$$

which can be solved iteratively [8]. This algorithm works across the whole image in the range and azimuth spatial frequency domains. As a result, the magnitudes in the images domain do not always balance perfectly. A final step is therefore required to adjust the means in the image domain. To demonstrate the channel balancing, Figs. 3 and 4 show a comparison of the coherency phase and intensity histograms for the 28 channels before and after channel balancing. The algorithm 
has clearly balanced the magnitude and phase, although there are a number of channels with quite low coherence. This is due to a mismatch in the hardware which has slightly offset the RF centre frequency between the up-chirp and down-chirp signals. While it is possible with post-processing to restrict the bandwidth to the common region and hence improve the coherency, the results in this paper have been generated using only the first 16 up-chirp phase centres.
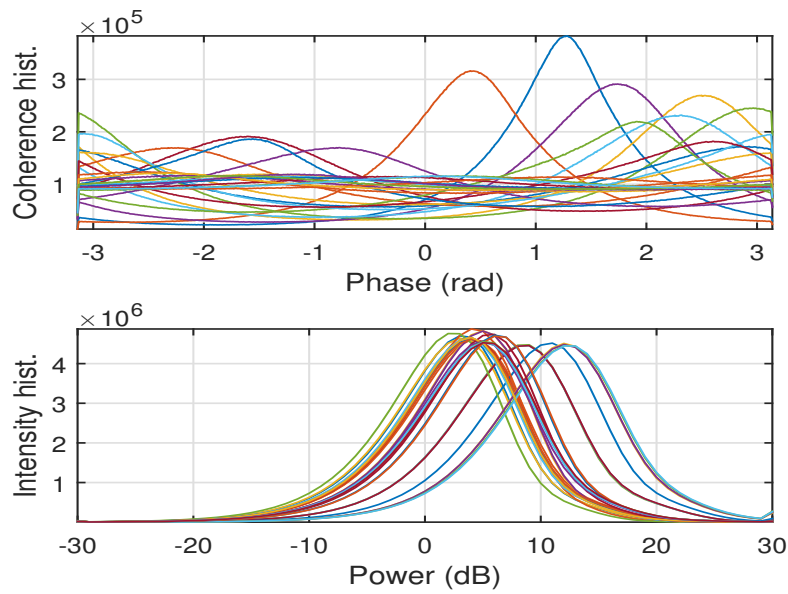

Fig. 3. Histograms of the channel coherency phase and intensity before channel balancing.
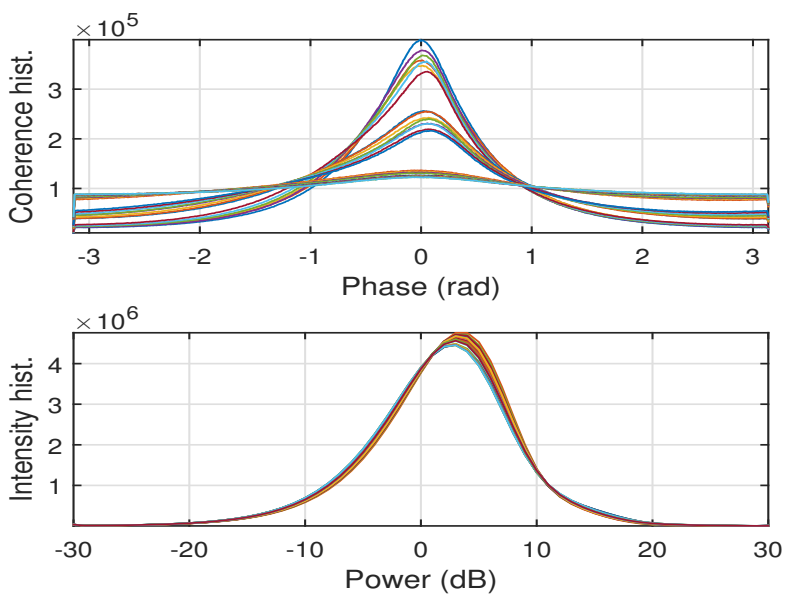

Fig. 4. Histograms of the channel coherency phase and intensity after channel balancing.

\section{SCEne Motion AnALysis}

In this paper, we illustrate the extraction of scene motion from the NRL MSAR data using the Velocity SAR (VSAR) algorithm [4]. VSAR provides the full Doppler spectrum at each and every pixel by means of a fast Fourier transform (FFT) down the 'time stack' of collocated images. Each image in this time stack is formed through standard SAR processing of data collected by a different phase center. Given the alongtrack spacing between the phase centers and the aircraft's along-track motion, these images can be adjusted to have slightly different collection times, but a common collection location in space. Thus, the phase progression down through the stack at any given pixel is related to the radial motion in the scene at that pixel. The FFT sorts the backscatter velocities present in each pixel, and transforms the time stack into a velocity stack, in which each image corresponds to a different radial velocity bin. If the backscatter in a given pixel is generated by multiple scatterers moving at different speeds, the Doppler spectrum of that pixel will exhibit multiple peaks, and thus energy will be found in multiple velocity images. This is in contrast to standard along-track interferometric SAR (ATISAR), in which a pair of collocated images provides a single velocity estimate at each pixel, namely, the pixel's Doppler centroid. While providing more detailed velocity information than ATI-SAR processing, VSAR's full motion spectrum can also be used to perform a much more accurate correction of distortions induced by the scene motion, one that does not leave behind 'holes' in the corrected imagery [5], [9].

The VSAR algorithm is illustrated with cartoons in Figs. 5 and 6, which depict MSAR images of a scene containing several vehicles (colored dots) traveling in the range direction along a smooth road (dark stripe). As shown in Fig. 5, the basic VSAR step is an FFT down the time stack at each pixel to transform the time stack into a velocity stack. The FFT sorts the backscatter into $M$ new images, according to radial Doppler velocity. In this cartoon, the red and yellow dots represent signatures of faster vehicles moving in opposite directions, while the blue and white dots correspond to slower vehicles moving in the same direction as the red. Variation in the brightness of the ambient area in the velocity stack images indicates that the surrounding clutter is basically stationary and thus most of the associated backscatter is sorted into the central (slow) velocity bins. This cartoon also depicts the 'train-offthe-track' distortion mechanism: the signatures of the radiallymoving vehicles do not lie on the signature of the road, in either the original time-stack or in the derived velocity images. This azimuthal displacement is proportional to the target's Doppler velocity, $v_{r}$, and is given by

$$
\delta_{a}=v_{r} R / v_{p}
$$

where $R$ is the distance to the object producing the backscatter and $v_{p}$ is the velocity of the SAR platform. In Fig. 5, the faster vehicles (red and yellow) are displaced more than those moving slowly (blue and white), and the vehicles traveling towards the radar (red, blue, white) are displaced in one direction, while those traveling on the opposite side of the road (yellow) are displaced in the other.

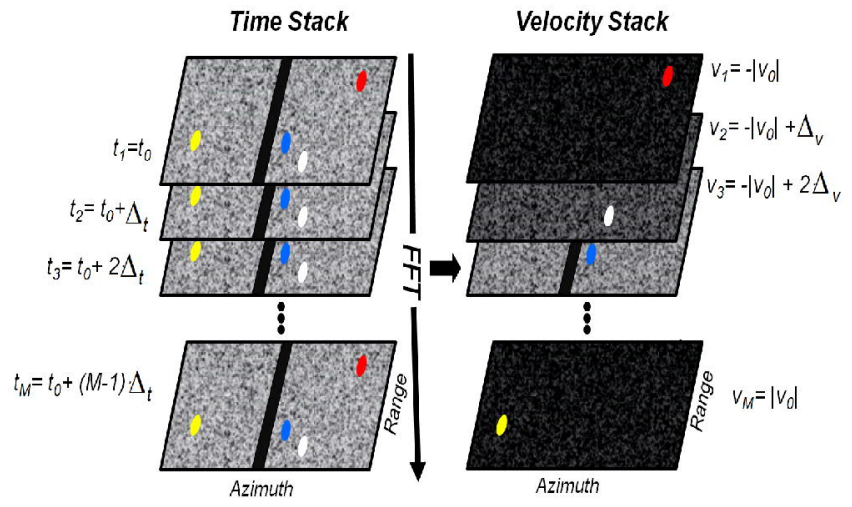

Fig. 5. Cartoon illustrating transformation of the image time-stack into a velocity stack through an FFT. Each colored dot represents a vehicle moving along a road (dark line). 


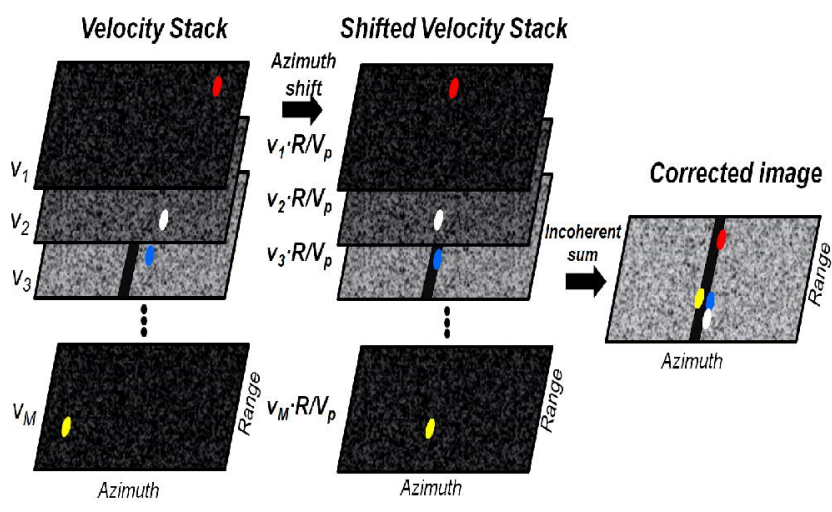

Fig. 6. Cartoon illustrating the VSAR corrective shifts.

While the VSAR motion spectrum is obviously useful in itself, it can also be used to correct the train-off-the-track distortions. This is depicted in Fig. 6. Through (5), the azimuth displacement error of each velocity image is known. These errors can therefore be corrected by simply shifting each velocity image back by this amount. As shown in the cartoon, this corrective shift places the signature of each vehicle in its proper position on the road. To form a single corrected image, the images in the shifted velocity stack can be summed incoherently. As demonstrated in [5], this same algorithm also corrects the more severe, smearing distortions suffered by target signatures characterized by a range of radial velocities, in particular, the signature of a marine vessel. However, this algorithm certainly has limitations [5]. The VSAR velocity resolution is inversely proportional to the length of the MSAR array, and thus fine resolution, required for a high-fidelity correction, requires a long array. Depending on the resolution requirements, the requisite array length may be impractical.

\section{INITIAL RESULTS}

The first flights of the NRL MSAR took place in September 2014 over several locations along the mid-Atlantic coast of the USA. Fig. 7 shows one of the test sites, Oregon Inlet on the Outer Banks of North Carolina. The scene includes Barrier Islands to the north and south of the inlet itself as well as a bridge span. This area was selected for flight testing due to the variety of moving targets in the scene, including vehicles, boats, tidal currents, and shoaling waves. For reference, an expanded view of the VSAR analysis region is shown in Fig. 8, which was produced by incoherently summing the 16 original SAR images. The corresponding VSAR image is then shown in Fig. 9 and is formed by incoherently summing the 16 shifted (i.e. corrected) velocity images.

As shown by a comparison of Figs. 8 and 9, VSAR processing corrects the smeared shoaling wave signatures (upper left side of image) by shifting the displaced backscatter contributions back to the actual location of the waves. The resulting signatures in Fig. 9 have a reduced width that is a more accurate measure of the actual size of the shoaling waves. There are also two faint echoes which have been highlighted and correspond to cars travelling on the bridge. These have been displaced in the imagery due to their radial velocities. The corrected VSAR image has then shifted these cars back towards the bridge, as shown by the highlighted regions in Fig. 9. As shown in the next section, these weak signatures can be enhanced in several ways.

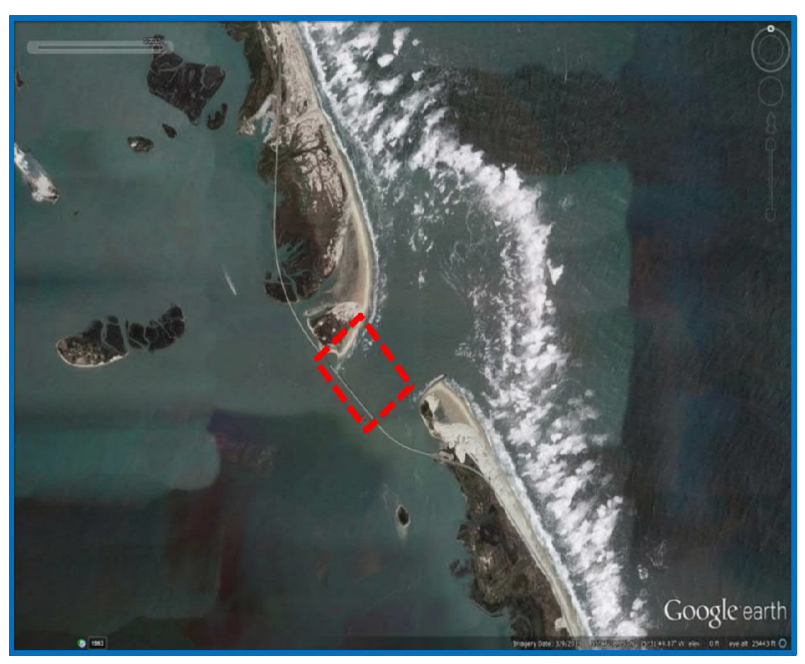

Fig. 7. Oregon Inlet test site. Red box indicates VSAR test region.

\section{VSAR ENHANCEMENT}

The VSAR results have demonstrated the potential for correcting for motion in SAR scenes. However, due to the noncoherent sum of 16 velocity images, some corrected targets appear quite faint in the VSAR image. By thresholding the magnitude in both the stationary and velocity images, any leakage of the stationary scatterers into the velocity stack can be removed and the dominant moving scatterers can be highlighted. This can be achieved by:

- Identify strong scatterers in the DC velocity image and mask those pixels in the other velocity images.

- Balance the means of the different velocity images relative to the DC velocity image.

- Mask non-significant scatterers in each non-DC velocity image.

Using these processing steps allows the strongest velocity scatterers to be highlighted through the velocity stack. Further improvement in the image quality and velocity resolution can be obtained by replacing the FFT with a fourth order Burg autoregressive spectral estimate. This model based approach was chosen due to the short length of the data sequence and since there are only a small number of significant velocity scatterers present in each pixel. The improved VSAR image using the spectral estimation algorithm with magnitude thresholding is shown in Fig. 10. Through experimentation, the threshold for masking strong scatterers was set as the mean of the DC image, while the significant scatterer threshold was set to 5 standard deviations above the mean for each image. Close inspection of this image now reveals a third target on the bridge which was obscured in the original VSAR image! Finally, if the strongest velocity magnitude is found for each pixel, the velocity profile can be overlayed onto the VSAR image. Fig. 11 shows the result: a composite VSAR image with greater contrast and the dominant velocity scatterers highlighted. Note that the two blue cars remain smeared over approximately $100 \mathrm{~m}$ in azimuth due to the cars accelerating up this section of the bridge. 


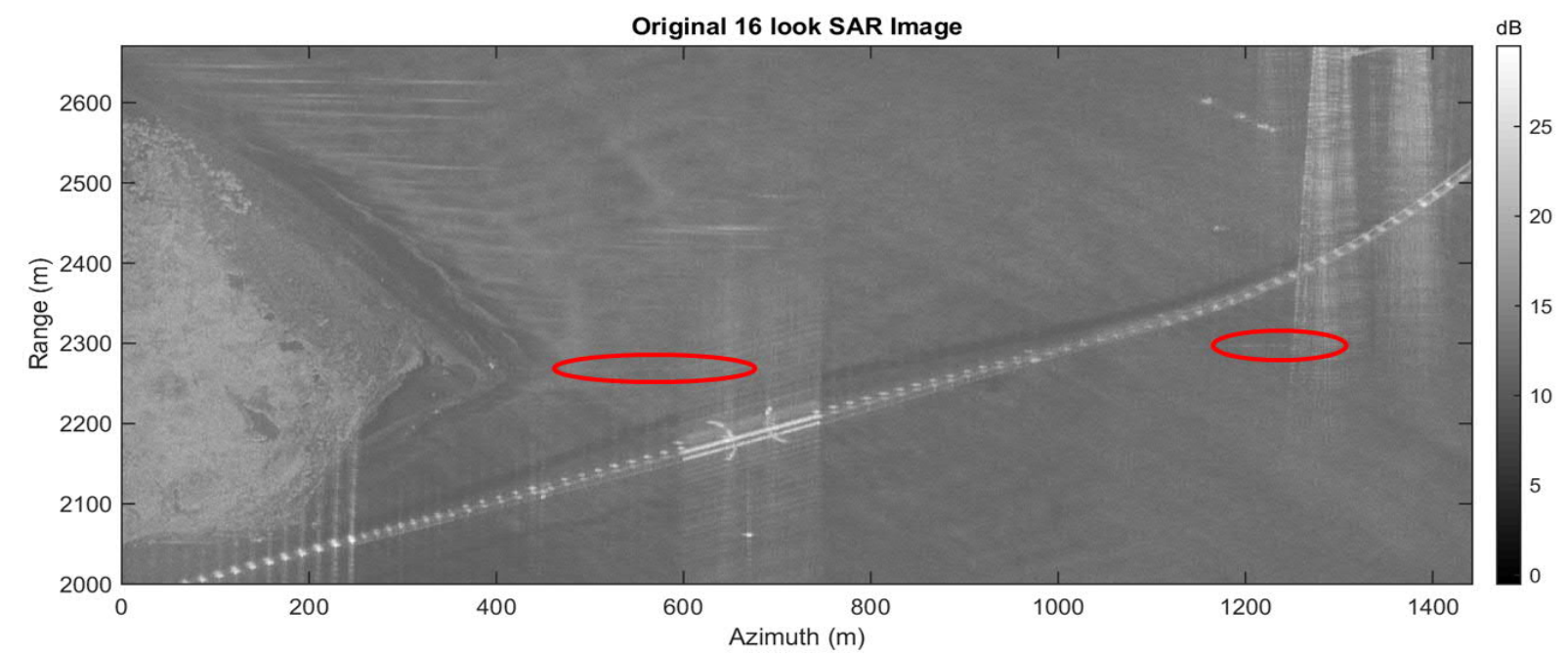

Fig. 8. Zoomed SAR image highlighting shifted cars.

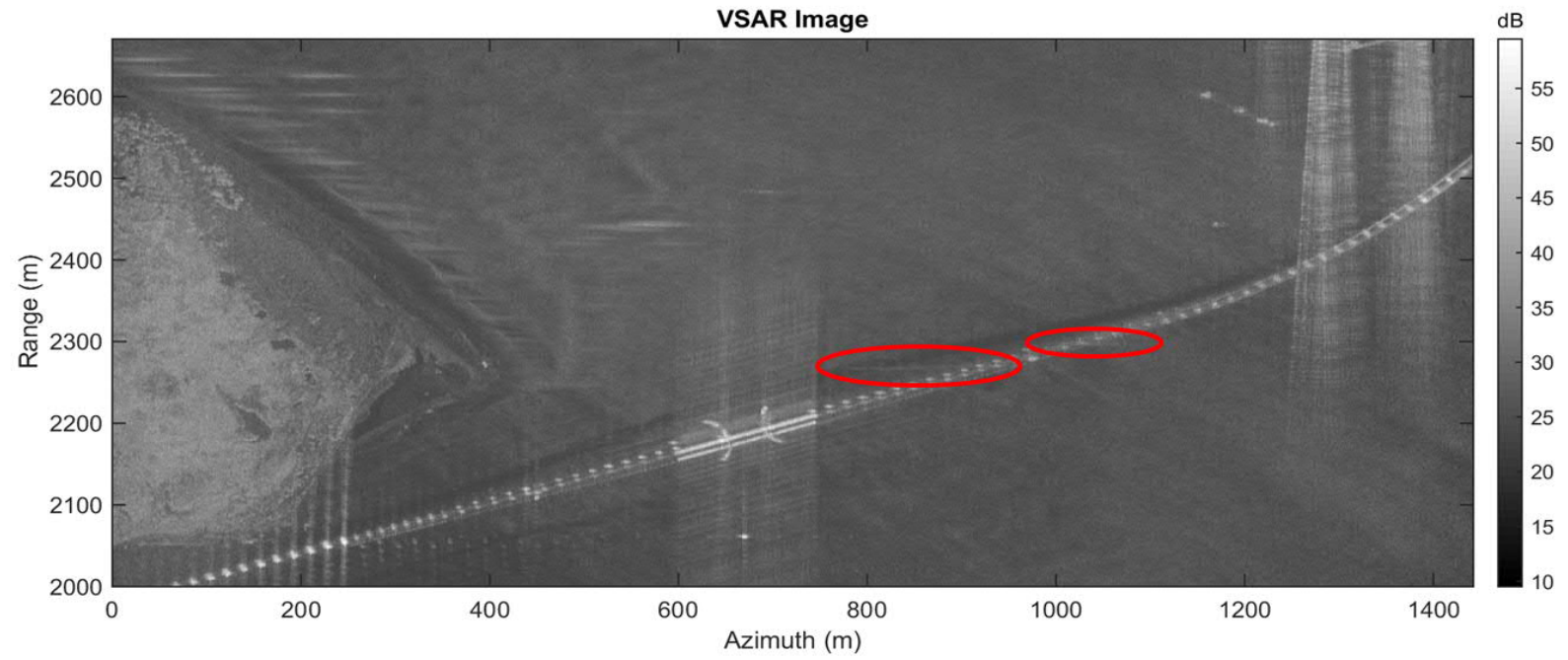

Fig. 9. VSAR image showing cars aligned with bridge.

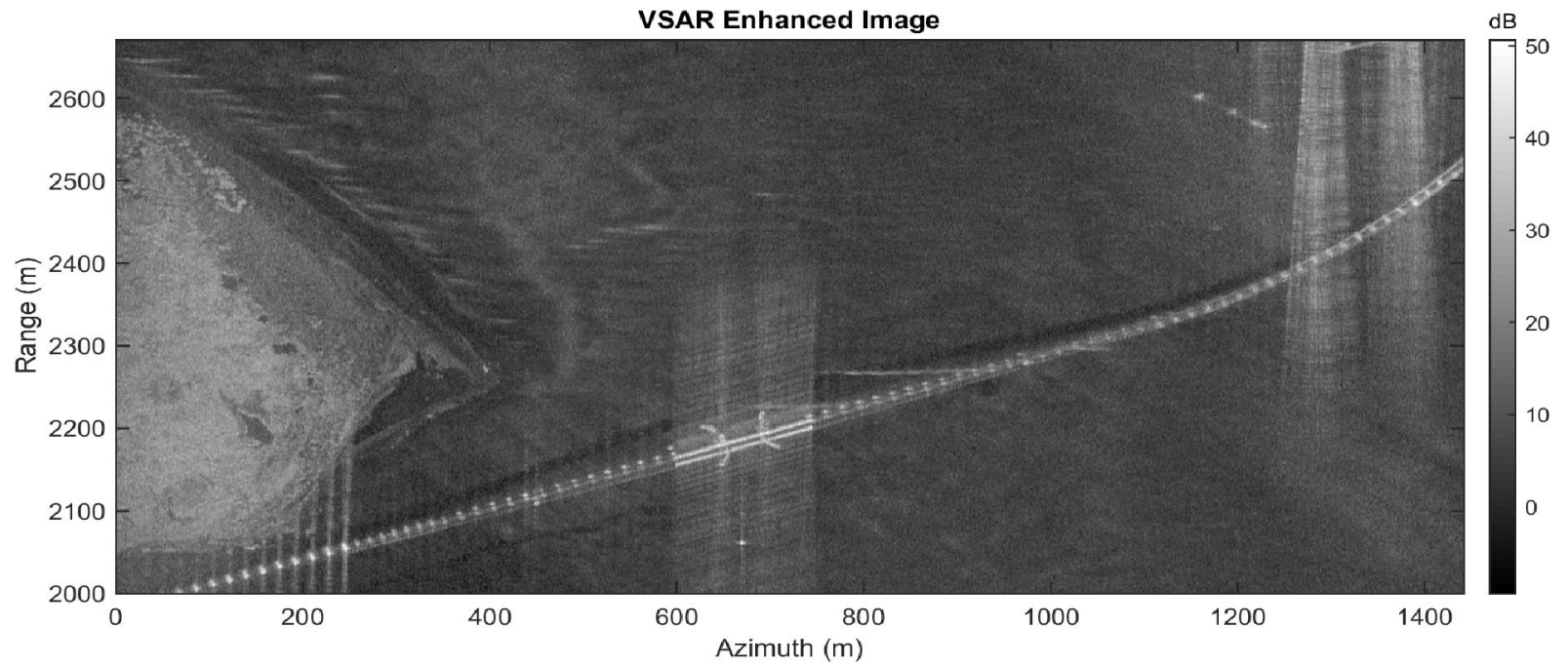

Fig. 10. Enhanced VSAR image using Burg AR spectral estimate and magnitude thresholding. 


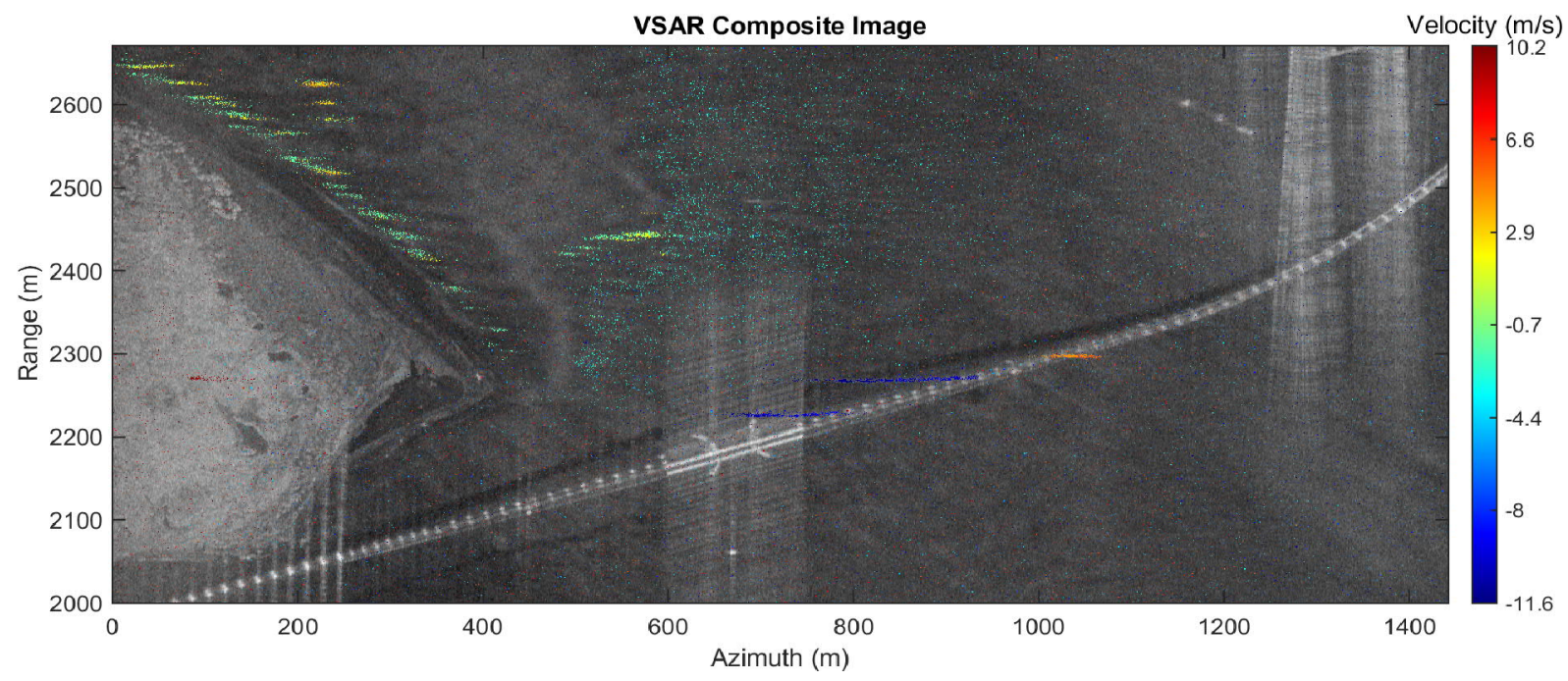

Fig. 11. Composite VSAR image highlighting dominant pixel velocity.

Even without processing enhancements such as these, alternate presentations of the basic VSAR data can be used to highlight weak signatures. This is illustrated in Fig. 12 which shows the shifted velocity stack for a subsection of the analysis region. For clarity, only the velocity images corresponding to $0, \pm 5.8$ and $\pm 8.7 \mathrm{~m} / \mathrm{s}$ are shown. This stack of images clearly shows the (stationary) bridge at $0 \mathrm{~m} / \mathrm{s}$ with the three moving scatterers clearly standing out at -5.8 and $+8.7 \mathrm{~m} / \mathrm{s}$. After accounting for the look direction of the radar relative to the bridge, these two radial velocities correspond to vehicle speeds of 48 and $64 \mathrm{mph}$, quite close to the posted speed limit of $55 \mathrm{mph}$. Fig. 12 also highlights another advantage of VSAR processing: while the vehicle signatures are weak in the standard SAR imagery (Fig. 8) and even in the corrected VSAR image (Fig. 9), they stand out clearly in their respective velocity images.

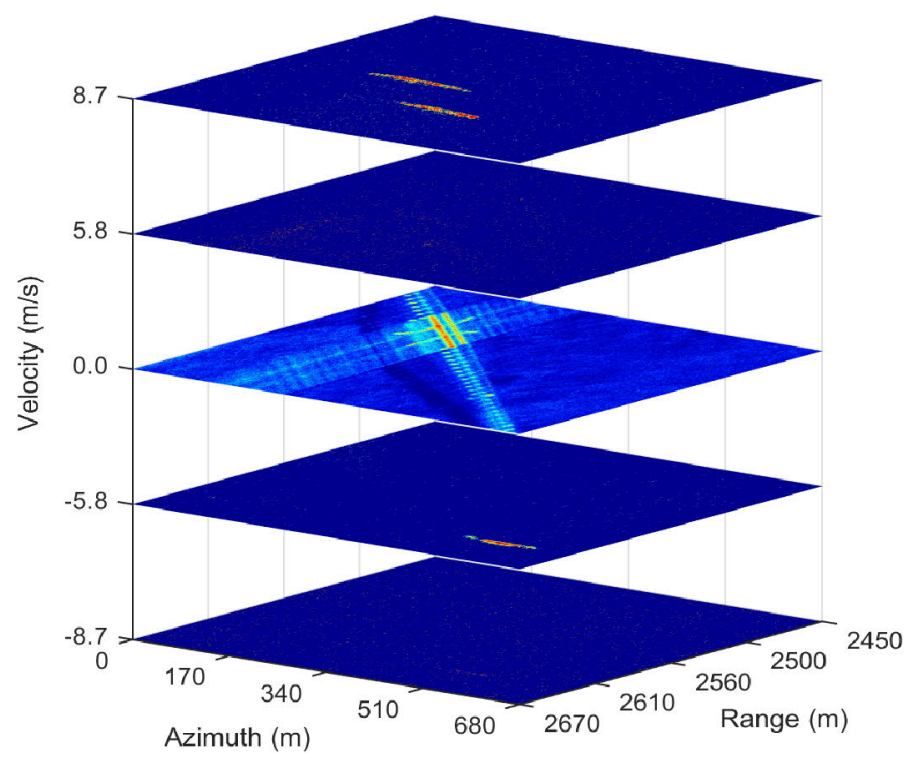

Fig. 12. VSAR image slice highlighting cars at -5.8 and $8.7 \mathrm{~m} / \mathrm{s}$.

\section{CONCLUSION}

This paper describes the NRL MSAR and presents initial results from the first deployment of the system. These results illustrate VSAR-based correction of azimuthal displacements of cars moving along a bridge over a tidal inlet, and also show the significant increase in detectability provided by the Doppler filtering inherent in VSAR processing. Future work is planned to improve the coherency across the 28 phase centres, investigate the velocity profiles of waves and boats and compare the VSAR performance against other adaptive techniques for clutter suppression.

\section{REFERENCES}

[1] K. Suwa, K. Yamamoto, M. Tsuchida, T. Wakayama, S. Nakamura, J. Endo, K. Hayashi, H. Hasegawa, and Y. Nakano, "An experimental study on image based multi-channel SAR-GMTI algorithm," in Asia-Pacific Conference on Synthetic Aperture Radar, Tsukuba, Japan, September 2013, pp. 577-580.

[2] A. Budillon, V. Pascazio, and G. Schirinzi, "Multi-baseline along track SAR interferometric systems for ground moving target indication," in International Geoscience and Remote Sensing Symposium, Honolulu, USA, July 2010, pp. 2924-2927.

[3] J. H. G. Ender, "Space-Time Processing for multichannel Synthetic Aperture Radar," Electronics and Communication Engineering Journal, vol. 11, no. 1, pp. 29-38, 1999.

[4] B. Friedlander and B. Porat, "VSAR: a high resolution radar system for ocean imaging," IEEE Transactions on Aerospace and Electronic Systems, vol. 34, no. 3, pp. 755-776, July 1998.

[5] M. A. Sletten, "Demonstration of SAR distortion correction using a ground-based multichannel SAR test bed," IEEE Transactions of Geoscience and Remote Sensing, vol. 51, no. 5, pp. 3181-3190, May 2013.

[6] A. Moreira, "Airborne SAR processing of highly squinted data using a chirp scaling approach with integrated motion compensation," IEEE Transactions on Geoscience and Remote Sensing, vol. 32, no. 5, pp. 1029-1040, September 1994.

[7] J. H. G. Ender, "The airborne experimental multi-channel SAR-system AER-II," in European SAR Conference, Königswinter, Germany, 1996, pp. $49-52$.

[8] C. H. Gierull, "Digital channel balancing of along-track interferometric SAR data," DRDC Ottawa, Technical Memorandum, March 2003.

[9] R. Romeiser, "Current measurement by airborne along-track InSAR: Measuring technique and experimental results," IEEE Journal of Oceanic Engineering, vol. 30, no. 3, pp. 552-569, 2005. 